\title{
Nuclear morphometry in histological specimens of canine prostate cancer: Correlation with histological subtypes, Gleason score, methods of collection and survival time
}

\author{
Guido Di Donato ${ }^{\mathrm{a}}$, Renée Laufer-Amorim ${ }^{\mathrm{b}}$, Chiara Palmieri ${ }^{\mathrm{c}}{ }_{*}$ \\ a Istituto Zooprofilattico Sperimentale dell'Abruzzo e del Molise "G. Caporale", Teramo, Italy \\ b Department of Veterinary Clinic, School of Veterinary Medicine and Animal Science - Univ. Estadual Paulista - UNESP, Botucatu, SP, Brazil \\ ' School of Veterinary Science, The University of Queensland, Gatton campus, Queensland, Australia
}

\section{A R T I C L E I N F O}

\section{Keywords:}

Dog

Nuclear morphometry

Prostate

Cancer

\begin{abstract}
A B S T R A C T
Ten normal prostates, 22 benign prostatic hyperplasia (BPH) and 29 prostate cancer (PC) were morphometrically analyzed with regard to mean nuclear area (MNA), mean nuclear perimeter (MNP), mean nuclear diameter (MND), coefficient of variation of the nuclear area (NACV), mean nuclear diameter maximum (MDx), mean nuclear diameter minimum (MDm), mean nuclear form ellipse (MNFe) and form factor (FF). The relationship between nuclear morphometric parameters and histological type, Gleason score, methods of sample collection, presence of metastases and survival time of canine PC were also investigated. Overall, nuclei from neoplastic cells were larger, with greater variation in nuclear size and shape compared to normal and hyperplastic cells. Significant differences were found between more (small acinar/ductal) and less (cribriform, solid) differentiated PCs with regard to FF ( $\mathrm{p}<0.05$ ). MNA, MNP, MND, MDx, and MDm were significantly correlated with the Gleason score of PC ( $\mathrm{p}<0.05$ ). MNA, MNP, MDx and MNFe may also have important prognostic implications in canine prostatic cancer since negatively correlated with the survival time. Biopsy specimens contained nuclei that were smaller and more irregular in comparison to those in prostatectomy and necropsy specimens and therefore factors associated with tissue sampling and processing may influence the overall morphometric evaluation.

The results indicate that nuclear morphometric analysis in combination with Gleason score can help in canine prostate cancer grading, thus contributing to the establishment of a more precise prognosis and patient's management.
\end{abstract}

\section{Introduction}

Cancer cells are notoriously characterized by nuclear changes, such as enlargement, variations in shape and modification of the chromatin pattern, which morphologically express the ongoing genetic and epigenetic changes occurring during carcinogenesis and the degree of nuclear differentiation (Sorensen, 1992). However, the evaluation of these nuclear alterations often lacks of objectivity and reproducibility (Baak et al., 1989). Therefore, the current trend in histopathology is to translate nuclear morphological changes into quantifiable features with digital image analysis through the quantitative nuclear morphometry.

Nuclear morphometry is significantly associated with histopathological grading of numerous types of cancer in humans (Bierhoff et al., 2003; Kontak and Campbell, 2003; Li et al., 2003) and it is considered an useful diagnostic predictor of recurrence and metastasis in different tumors in animals, such as mast cell tumor (Maiolino et al., 2005; Strefezzi et al., 2003), cutaneous squamous cell carcinoma (Maiolino et al., 2002), seminoma (Maiolino et al., 2004), mammary tumors (Ciurea et al., 1992; De Vico and Maiolino, 1997; Destexhe et al., 1995; Simeonov and Simeonova, 2007), acanthomatous ameloblastoma (Martano et al., 2006), anal sac (Simeonov and Simeonova, 2008) and melanocytic tumors (Roels et al., 2000).

Despite several studies demonstrating the value of the morphometric methods in prognosis and as indicators of malignant potential of human prostate cancer (PC) (Diamond et al., 1982; Kavantzas et al., 2001; Partin et al., 1992), a detailed nuclear morphometric analysis has never been reported in canine prostate cancer.

A standardized histopathological classification of canine PC in order to identify the degree of differentiation or grading is currently lacking, although a subjective histological assessment may be based on different

\footnotetext{
* Corresponding author at: School of Veterinary Science, The University of Queensland, Gatton campus 4343, Gatton, QLD, Australia.

E-mail address: c.palmieri@uq.edu.au (C. Palmieri).
} 
parameters, such as invasiveness, cellular morphology and mitotic index whose prognostic usefulness is however yet to be validated. Compared to human PC, the Gleason grading system, although applied in the canine scenario (Palmieri and Grieco, 2015), is not traditionally accepted and validated for grading canine prostatic carcinomas and even when considered in men, Gleason grading may be biased by interobserver and intraobserver variations. When using the revised version for Gleason scoring, estimated interobserver reproducibility is $>80 \%$ (Melia et al., 2006). However, reproducibility remains critical in all grading systems due to the subjective semiquantitative evaluation of the slides and the different degree of pathology training. Quantitative nuclear morphometry has therefore several advantages, such as obtaining objective and reproducible measurements, detecting not obvious nuclear changes, being potentially applicable to small tissue specimens and allowing fine numerical results to be statistically analyzed so that the probability level of a classification based on quantitative data can be calculated.

Many studies have demonstrated that a computer-based nuclear morphometry system can refine the prognostic information provided by the Gleason scoring system (Hurwitz et al., 1999; Khan et al., 2003; Zhang et al., 2000). Additionally, the World Health Organization (WHO) recommends to use nuclear morphometry in prostate cancer (Epstein et al., 2004).

The aim of the present study was to investigate whether quantitative measurements of nuclear variables could be used as objective diagnostic and, if possible, prognostic criteria for prostate cancer in dogs. We evaluated the relationship between nuclear morphometric features and histological type, Gleason score, metastases, type of sample collection, and survival time with the purpose to evaluate whether morphometric parameters may be useful in predicting the biological behavior of canine PC or distinguishing between certain histological subtypes of PC, as well as whether different methods of sample collection may have profound implications in the final morphometric evaluation.

\section{Materials and methods}

\subsection{Samples}

Sixty-one prostatic samples were examined. All samples were formalin-fixed, and paraffin wax-embedded, and sections $(4 \mu \mathrm{m})$ were stained with hematoxylin and eosin (HE). These included 10 normal prostates, 22 benign prostatic hyperplasia (BPH) and 29 prostate carcinomas (PC). PCs were classified according to the growth patterns described by the human WHO classification of Tumors of the Urinary System and Male Genital Organs (Epstein et al., 2004) and adapted to canine PCs (Palmieri et al., 2014). Based on this classification system, the following growth patterns were identified: cribriform (14/29), solid $(9 / 29)$ and small acinar/ductal (6/29). PC samples were further classified according to the Gleason grading - the most useful prognostic predictor of prostate cancer in men (Gleason, 1966) - that has been recently updated by the International Society of Urological Pathology (Epstein et al., 2016) and further applied in dogs (Palmieri and Grieco, 2015). Gleason grading assigns numerical grade (Ali et al., 2001; Baak, 1983; Baak et al., 1989; Bektas et al., 2009; Bierhoff et al., 2003) based on the architectural pattern of the tumor with a progressive lack of the differentiated acinar pattern from Grade 1 to Grade 5. Grades 1, 2 and 3 represent tumors that most closely resemble normal prostate gland, while Grades 4 and 5 are tumors showing increasingly abnormal glandular architecture (Gleason, 1966; Palmieri and Grieco, 2015). A primary grade is assigned to the most prevalent pattern, while the second most prevalent pattern is the secondary grade. The final Gleason score is then obtained by adding the primary and secondary grades together. If there is only one pattern, its grade is simply doubled to reach the score. Based on the Gleason system, canine PCs were graded as Grade $5(21 / 29)$, Grade $4(5 / 29)$ and Grade $3(3 / 29)$. The final Gleason score (GS) was as follows: GS10 (18/29), GS9 (7/29), GS8 (4/
29).

PC specimens were represented by tissues samples collected during necropsy $(n=13)$, prostatectomy $(n=5)$ or biopsy (by ultrasound or explorative laparotomy; $\mathrm{n}=8$ ).

In 3 cases, the information about the type of sample was not available.

Metastases were observed in 10 cases.

Survival times were available only in 8 cases (all GS10), ranging from 42 to 320 days.

\subsection{Morphometric analysis}

Nuclear morphometric analysis was performed on HE-stained sections by means of a microscope (Olympus) connected to a digital camera system and a computer equipped for image analysis (Image Pro Plus Program). For each specimen, 10 images of cell fields with a $40 \times$ objective lens were randomly captured, encompassing all cellular areas of the slide.

A total of 100 nuclei from intact cells that were not superimposed and had sharp nuclear limits were traced with the help of a computer mouse. All areas with evidence of hemorrhage, necrosis, inflammation, surgical crush injury and fixation artifact were excluded.

All tracings were conducted by a single observer to minimize interobserver variance.

The following parameters were measured (Fig. 1): mean nuclear area $\left(\mathrm{MNA}, \mu \mathrm{m}^{2}\right.$ ), mean nuclear perimeter (MNP, $\left.\mu \mathrm{m}\right)$, coefficient of variation of the nuclear area (NACV) (SD/MNAx100), mean nuclear diameter (MND; $\mu \mathrm{m})$, mean nuclear diameter maximum (MDx, $\mu \mathrm{m})$, mean nuclear diameter minimum (MDm, $\mu \mathrm{m})$. NACV was calculated to express variations in size in individual cases.

From these basic data (direct parameters), so-called form factors were derived. They are nonparametric measures and quantitatively express the shape of a structure. The form factors selected in this study aimed to determine the deviation of a perfect circle of a nuclear section examined. The following form factors were derived: mean nuclear form factor or roundness factor (perimeter ${ }^{2} / 4 \pi \times$ area) (FF) and mean nuclear form ellipse or LS ratio (longest diameter/shortest diameter) (MNFe).

FF is a measure of nuclear roundness and represents a dimensionless size-invariant shape descriptor that yields a minimal value of 1.00 for a perfect circle and increase as the shape of a contour deviates from circularity. In nuclear morphometry, roundness is indicative of a regular nuclear morphology such as that of a normal cell or a benign proliferative condition. FF is therefore reflective of the degree of deviation from a perfect circle of the 2D binary shape of a nucleus.

$\mathrm{MNFe}$ is a measure of ellipticity and it corrects the nuclear roundness determination for smooth elliptical nuclei. In a round circle, MNFe values correspond to 1 ; if the object is elliptical, the MNFe is $>1$.This shape descriptor is a reliable tool to assess the change in shape and outline of the nucleus.

\subsection{Statistical analysis}

A statistical analysis was carried out to study the significance of differences of the morphometric parameters (MNA, MNP, NACV, MDx, $\mathrm{MDm}, \mathrm{FF}, \mathrm{MNFe}$ ) between normal prostates, BPH and PC, as well as histological types, tumor grading and type of sample. The nuclear parameters between all the groups overall were compared using Analysis of variance (ANOVA) and between the individual groups using the post hoc Bonferroni-Holm Multiple Comparisons Test. A $t$-test was used to evaluate the differences between metastatic and non-metastatic lesions. The relationship between each nuclear parameter and survival time was verified by the Spearman correlation using GraphPad Prism. The accepted level of significance was $\mathrm{p}<0.05$. 


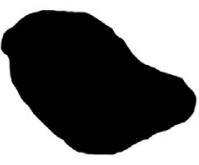

Nuclear area

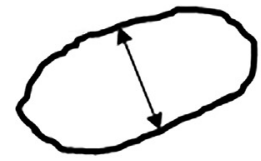

Minimum nuclear diameter

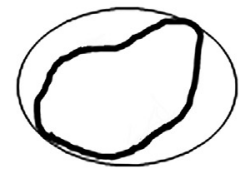

Form factor

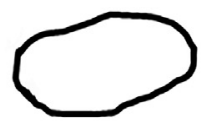

Nuclear perimeter

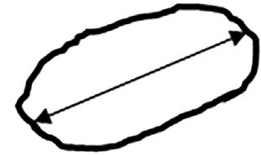

Maximum nuclear diameter

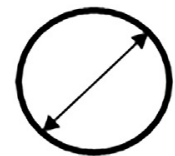

Nuclear diameter

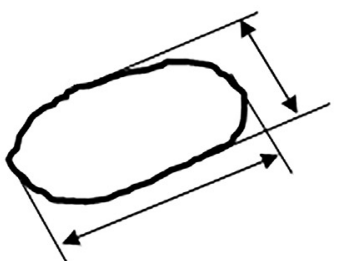

Nuclear form ellipse (LS ratio)

\section{NACV (coefficient of variation of the nuclear area): standard deviation of nuclear area \\ mean nuclear area}

Fig. 1. Illustration of parameters for quantitative estimation of nuclear morphometry in canine prostatic lesions

\section{Results}

The mean values of the nuclear morphometric parameters in normal prostates, BPH and PC are given in Table 1; and those for tumors of different histological subtype, Gleason grade, Gleason score, type of collection and association with metastases are given in Table 2.

A statistically significant increase of MNA (normal vs. BPH, BPH vs. $\mathrm{PC}=\mathrm{p}<0.01$; normal vs. $\mathrm{PC}=\mathrm{p}<0.05$ ), MNP (normal vs. $\mathrm{PC}$, $\mathrm{BPH}$ vs. $\mathrm{PC}=\mathrm{p}<0.01$; normal vs. $\mathrm{BPH}=\mathrm{p}<0.05$ ), MND (normal vs. $\mathrm{PC}, \mathrm{BPH}$ vs. $\mathrm{PC}=\mathrm{p}<0.01$; normal vs. $\mathrm{BPH}=\mathrm{p}<0.05)$, $\mathrm{MDx}$ (normal vs. BPH, BPH vs. $\mathrm{PC}=\mathrm{p}<0.01$; normal vs. $\mathrm{PC}=\mathrm{p}<0.05$ ) and MDm ( $p<0.01)$ was observed from normal to hyperplastic and neoplastic prostates. Moreover, PCs showed statistically significant higher NACV $(\mathrm{p}<0.01)$ and MNFe $(\mathrm{p}<0.01)$ compared to normal prostates and $\mathrm{BPH}$, while no differences in $\mathrm{FF}$ were revealed between the three conditions, although a slightly higher value has been observed in PCs compared to normal prostates and BPH.

Solid and cribriform PCs were characterized by significant higher values of FF compared to small acinar/ductal PCs ( $p<0.05$ ). NACV, $\mathrm{MNFe}$ and FF were higher in the cribriform compared to the other two histological subtypes of PCs, although there was no statistical difference. MNA, MNP, MND, MDx, MDm were higher in solid PC compared to cribriform and small acinar/ductal PC, although the differences were not statistically significant.

No statistically significant differences were observed between the three Gleason grades, although Grades 5 and 4 were characterized by higher MNA, MNP, NACV, MND, MDx, MDm, MNFe and FF compared to Grade 3.

Regarding the final Gleason score, MNA ( $\mathrm{p}<0.01$ ), MNP ( $\mathrm{p}<0.01$ ), MND ( $\mathrm{p}<0.01$ ), MDx (GS10 vs. GS8 $=\mathrm{p}<0.01$; GS9 vs. GS8 $=\mathrm{p}<0.05)$ and MDm (GS10 vs. GS8 $=\mathrm{p}<0.01$; GS9 vs. GS8 $=p<0.05$ ) were higher in G9 and G10 compared to G8. NACV and FF were higher in GS10 compared to GS9 and GS8, although the differences were not statistically significant. MNFe was higher in GS9 compared to GS10 and GS8, although the differences were not statistically significant.

Samples collected during necropsies showed a significant higher MNA compared to biopsies $(\mathrm{p}<0.05)$. Samples collected during necropsy showed a significant higher MNP compared to the other two groups $(\mathrm{p}<0.01)$.
Although non metastatic lesions were characterized by higher MNA, MNP, NACV, MND, MDm, MNFe and FF compared to metastatic tumors, the only parameter with a statistically significant difference was MDx $(\mathrm{p}<0.05)$.

The values of each nuclear parameter in the 8 cases with different survival times are summarized in Table 3 and Fig. 2.

The neoplastic cell MNA, MND, MDx and MNFe and survival times were significantly correlated as evidenced in Table 4 .

\section{Discussion}

Computerized image analysis allows accurate and objective evaluation of nuclear morphology and it has been used to show that increases in nuclear size and irregularity of nuclear shape are more frequently detected in malignant tumors than nonmalignant borderline tumors.

The present results demonstrate that area, mean diameter and ellipticity of the nucleus are the most appropriate nuclear morphometric parameters for differentiating between PCs with different survival times. The same parameters, including the nuclear perimeter (MNP), may be also useful to identify different histopathological grades.

In our study, we observed an increased size of the nuclei proceeding from normal to hyperplastic to neoplastic prostate, most likely reflecting an increase in DNA synthesis and cell proliferative activity. In a previous study of advanced human gastric cancer there was a significant correlation between nuclear area of cancer cells and expression of p53, Ki67 labelling index and DNA ploidy of the tumors (Ikeguchi et al., 1999). Human hepatocellular carcinoma cells with large nuclear area have more chances to invade blood vessels resulting in intrahepatic and distant metastases compared to neoplastic cells with small nuclear area (Ikeguchi et al., 1998). Moreover, as expected, cells from malignant lesions have more irregular nuclear shapes than normal and hyperplastic epithelial cells and this may be considered an indicator of genetic instability and intratumoral heterogeneity (Gisselsson et al., 2001).

In addition, we observed an increase of the nuclear area and perimeter in less differentiated tumors (solid and cribriform PCs) that may reflect the different degree of cell differentiation and proliferative activity of neoplastic cells.

Regarding the Gleason grade, significant alterations in the nuclear 

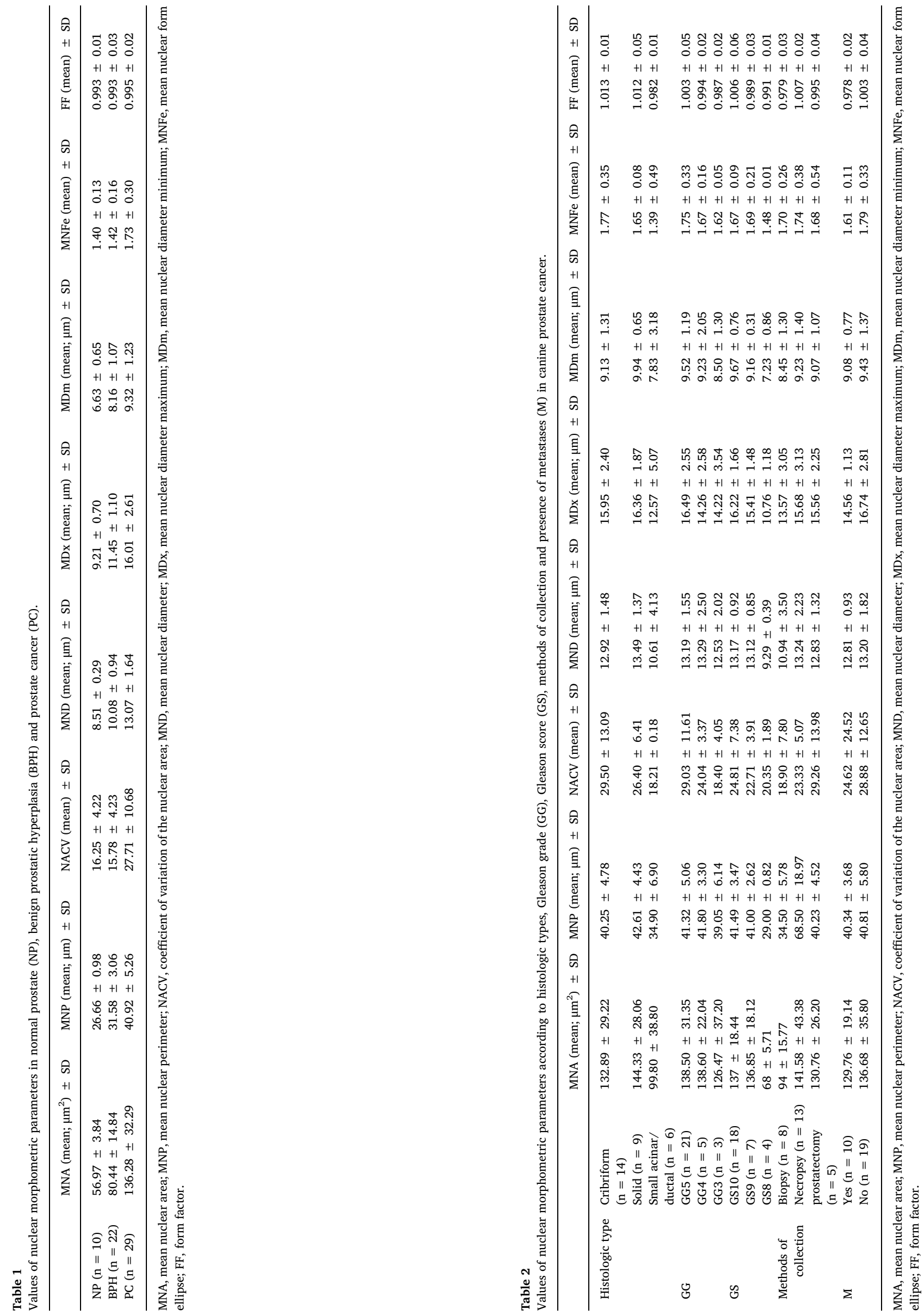
Table 3

Values of nuclear morphometric parameters in the 8 cases of canine prostate cancer with different survival rates.

\begin{tabular}{|c|c|c|c|c|c|c|c|c|}
\hline & Case no. 1 & Case no. 2 & Case no. 3 & Case no. 4 & Case no. 5 & Case no. 6 & Case no. 7 & Case no. 8 \\
\hline Survival (days) & 87 & 42 & 220 & 160 & 320 & 130 & 55 & 190 \\
\hline $\operatorname{MNA}\left(\mu \mathrm{m}^{2}\right)$ & 151.74 & 168.60 & 139.10 & 94.02 & 107 & 129.05 & 174 & 127.10 \\
\hline $\operatorname{MNP}(\mu \mathrm{m})$ & 46.41 & 46.96 & 41.54 & 34.20 & 36 & 40.05 & 46.10 & 40 \\
\hline NACV & 0.24 & 0.23 & 0.23 & 0.34 & 0.75 & 0.34 & 0.27 & 0.26 \\
\hline $\operatorname{MND}(\mu \mathrm{m})$ & 13.90 & 14.65 & 13.31 & 10.94 & 11.67 & 12.82 & 14.89 & 12.72 \\
\hline $\operatorname{MDx}(\mu \mathrm{m})$ & 9.71 & 11.17 & 9.51 & 7.49 & 1.08 & 9.97 & 11.23 & 9.17 \\
\hline $\operatorname{MDm}(\mu \mathrm{m})$ & 16.20 & 16.82 & 18.37 & 12.57 & 17.59 & 16.19 & 19.52 & 16.16 \\
\hline MNFe & 1.67 & 1.50 & 1.93 & 1.68 & 2.89 & 1.62 & 1.74 & 1.76 \\
\hline $\mathrm{FF}$ & 1.130 & 1.041 & 0.9898 & 0.979 & 0.964 & 0.988 & 0.968 & 1.003 \\
\hline
\end{tabular}

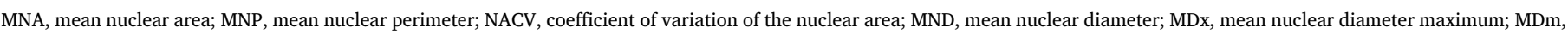
mean nuclear diameter minimum; MNFe, mean nuclear form ellipse; FF, form factor.

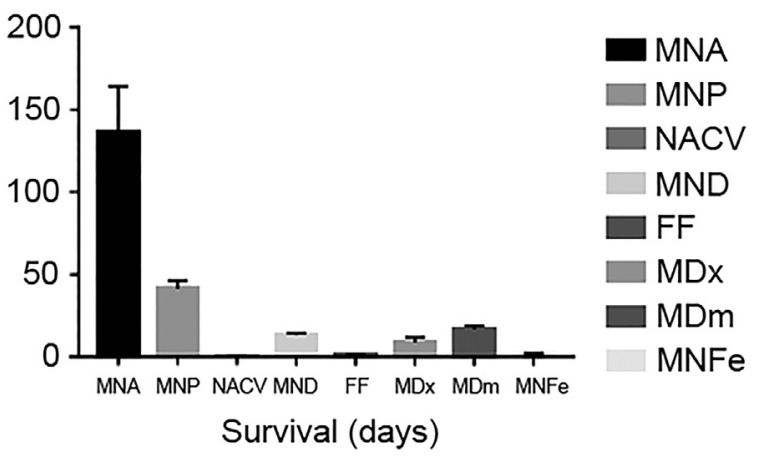

Fig. 2. Nuclear morphometric parameters and survival rates (days) in eight cases of canine prostatic carcinoma. Tumors with less survival rates have a higher MNA, MND, $\mathrm{MDx}$ and MNFe compared to tumors with high survival rates $(\mathrm{p}<0.05)$.

Table 4

Correlation coefficient (r) between the survival time and nuclear morphometric parameters.

\begin{tabular}{lll}
\hline & Spearman $\mathrm{r}$ & p value \\
\hline Survival (days) vs. MNA & -0.74 & $0.046^{*}$ \\
Survival (days) vs. MNP & -0.72 & 0.052 \\
Survival (days) vs. NACV & 0.45 & 0.267 \\
Survival (days) vs. MND & -0.74 & $0.046^{*}$ \\
Survival (days) vs. MDx & -0.86 & $0.010^{*}$ \\
Survival (days) vs. MDm & -0.06 & 0.898 \\
Survival (days) vs. MNFe & 0.83 & $0.015^{*}$ \\
Survival (days) vs. FF & -0.44 & 0.273 \\
\hline
\end{tabular}

MNA, mean nuclear area; MNP, mean nuclear perimeter; NACV, coefficient of variation of the nuclear area; MND, mean nuclear diameter; MDx, mean nuclear diameter maximum; $\mathrm{MDm}$, mean nuclear diameter minimum; MNFe, mean nuclear form ellipse; FF, form factor.

* Statistically significant $(\mathrm{p}<0.05)$

structure have been described by Veltri et al. (2007) between and within Gleason grading patterns 3/4 and 4/5. Recently, Ali et al. (2001) using a total of seven nuclear features could differentiate Gleason grade patterns 3 vs. 4 with a classification accuracy of $84 \%$. However, in our cases, none of the nuclear parameters were significantly correlated with the Gleason grade.

On the other hand, when considering the overall Gleason score of canine PC, a significant correlation with MNA, MNP, MND, MDx, and MDm was detected.

Quite interestingly, survival time has a significant inverse correlation with MNA, MNS, MDx and MNFe in canine prostate cancer, matching similar results in human PC (Bektas et al., 2009; Choi et al., 1999; Martinez-Jabaloyas et al., 2002; Zhang et al., 2000).

No significant differences were observed between metastatic and non-metastatic canine PCs, as already reported by Diamond et al. (1982) in men with a mean nuclear area of $31 \mu \mathrm{m}^{2}$ and $30.7 \mu \mathrm{m}^{2}$ for those patients who were free of metastases post-operatively and those with metastases. Our findings may suggest that canine PCs are generally malignant or potentially malignant since anaplastic features occur regardless of the presence or absence of metastases. Nuclear parameters should therefore not necessarily considered potential predictors of metastases in canine PCs.

Finally, we compared nuclear morphometry on needle biopsy, prostatectomy and necropsy specimens to determine whether any of those methods of sample collection may affect nuclear parameters and values. Nuclear morphometry from biopsy specimens revealed nuclei that were smaller and more irregular in comparison to those in prostatectomy or necropsy specimens.

Altered nuclear features may be attributed to compression artifacts from the passage of the needle through the prostatic tissue, although our biopsies were devoid of any microscopic signs of tissue trauma. Moreover, nuclear injury should occur at the edge of the biopsy specimen and not diffusely throughout it.

Other possible factors would be the different impact of tissue shrinkage during the histological processing between biopsy and surgical specimens (Baak et al., 1989) or the intratumoral heterogeneity (Fujikawa et al., 1997). Limited amount of tissue is retrieved with biopsy and it is not reflective of the wide range of size and shape that may exist in the same tumor, which is otherwise better represented in prostatectomy or necropsy samples. However, it is most likely that a delayed fixation and/or excessive air drying of the tissue may influence the nuclear parameters (Baak et al., 1989; Serratosa et al., 1988). In previous studies, the average values for the mean nuclear volume, mean nuclear area and form factor of neoplastic prostatic cells in biopsy samples were significantly smaller than those within prostatectomy specimens (Mohler et al., 1994; Zhang et al., 1999), as in our cases. Voges et al. (1992) reported an increased hepatocyte nuclear volume due to delayed fixation of rat liver, most likely caused by the continuous transcriptional activity occurring between collection and fixation and leading to an artefactual rounding of the nuclei. This may also apply to our prostatic samples if we consider that in clinical and necropsy practice, a time lag exists between prostate removal and start of the fixing procedure compared to a rapid fixation of biopsy samples. Therefore nuclear morphometry performed on biopsy specimens more accurately reflects reality than does analysis of prostatectomy and necropsy samples. Alternatively, an immediate start and rapid completion of fixation may be desirable to preserve the nuclear morphology of non-biopsy specimens.

Based on our findings, we suggest that MNA, MNP, MND, NACV and $\mathrm{MNFe}$ may be considered appropriate morphometric parameters for differentiating benign from malignant canine prostatic lesions in histological specimens, while MNA, MNP, MND, MDx and MDm should be used to differentiate PCs with different Gleason scores. MNA, MNS, $\mathrm{MDx}$ and MNFe may also have important prognostic implications in canine prostate cancer. Moreover, factors associated with tissue sampling and processing, which are not specific to cancer histologic 
features, should be considered before approaching any morphometric analysis since they have a significant influence on the nuclear size and shape of prostate cells.

We suggest that morphometric analysis, combined with a Gleason scoring system and ideally prognostic molecular markers, may be used in grading tumor malignancy and classifying borderline lesions in the routine clinical setting. Ancillary computerized morphometry can be complementary to the histopathological examination by providing several numerical parameters with increased objectivity and it might improve our understanding of the diagnostic and prognostic features of canine PC.

The results do not permit as of now a precise classification of PCs in terms of prognosis since the data concerning the evolution of this tumor have been analyzed in a small number of cases. However, these preliminary results may allow the establishment of fixed minimum and maximum values for each parameter investigated in order to classify PCs in a rapid and precise manner in terms of degree of differentiation which makes this a method of great interest to the pathologists. Once the diagnosis of PC is made by histopathology, tumor grading should include nuclear morphometry in order to predict patient's outcome and survival rate. Clearly the potential for applying quantitative histomorphometry and nuclear morphometry as new "biomarkers" will require further validation.

\section{Acknowledgements}

The authors thank CNPq (Brazilian National Council for Scientific and Technological Development) for the financial support for RLA (grant 306055/2011-2012 and 443884/2014-2015).

\section{References}

Ali, S., Veltri, R., Epstein, J.I., Christudass, C., Madabhushi, A., 2001. Adaptive energy selective active contour with shape priors for nuclear segmentation and gleason grading of prostate cancer. Med. Image Comput. Assist. Interv. 14 (Pt 1), 661-669.

Baak, J.P.A., 1983. Basis of morphometry. In: Baak, J.P.A., Oort, J. (Eds.), A Manual of Morphometry in Diagnostic Pathology. Springer-Verlag, Berlin-Heidelberg-New York, pp. 5-39.

Baak, J.P., Noteboom, E., Koevoets, J.J., 1989. The influence of fixatives and other variations in tissue processing on nuclear morphometric features. Anal. Quant. Cytol. Histol. 11, 219-224.

Bektas, S., Bahadir, B., Dogan Gun, B., Kertis, G., Ozdamar, S.O., 2009. The relation between gleason score, and nuclear size and shape factors in prostatic adenocarcinoma. Turk. J. Med. Sci. 39 (3), 381-387.

Bierhoff, E., Appel, K., Appel, T., Buettner, R., Wardelmann, E., 2003. Comparative morphometric analysis of primary versus recurrent basal cell carcinoma and of histological subtypes, significance of morphometry of the nuclei. Anticancer Res. 23, 2697-2700.

Choi, N.G., Sohn, J.H., Park, H.W., Jung, T.Y., 1999. Apoptosis and nuclear shapes in benign prostate hyperplasia and prostate adenocarcinoma: comparison with and relation to Gleason score. Int. J. Urol. 6 (1), 13-18.

Ciurea, D., Wilkins, R.J., Shalev, M., Liu, Z., Barba, J., Gil, J., 1992. Use of computerized interactive morphometry in the diagnosis of mammary adenoma and adenocarcinoma in dogs. Am. J. Vet. Res. 53 (3), 300-303.

De Vico, G., Maiolino, P., 1997. Prognostic value of nuclear morphometry in feline mammary carcinomas. J. Comp. Pathol. 117, 99-105.

Destexhe, E., Bicker, E., Coignoul, F., 1995. Image analysis evaluation of ploidy, S-phase fraction and nuclear area in canine mammary tumours. J. Comp. Pathol. 113 (3), 205-216.

Diamond, D.A., Berry, S.J., Umbricht, C., Jewett, H.J., Coffey, D.S., 1982. Computerized image analysis of nuclear shape as a prognostic factor for prostatic cancer. Prostate 3 , 321-332.

Epstein, J.I., Helpap, B., Algaba, F., et al., 2004. Tumours of the prostate. In: Eble, J.N., Sauter, G., Epstein, J.I., Sesterhenn, I.A. (Eds.), World Health Organization Classification of Tumors. Pathology and Genetics of Tumours of the Urinary System and Male Genital Organs. IARC Press, Lyon, pp. 159-192.

Epstein, J.I., Egevad, L., Amin, M.B., Delahunt, B., Srigley, J.R., Humphrey, P.A., 2016. The 2014 International Society of Urological Pathology (ISUP) consensus conference on gleason grading of prostatic carcinoma: definition of grading patterns and proposal for a new grading system. Am. J. Surg. Pathol. 40 (2), 244-252.

Fujikawa, K., Sasaki, M., Aoyama, T., Itoh, T., Yoshida, O., 1997. Intratumoral heterogeneity in prostate cancer demonstrated by volume-weighted mean nuclear volume. APMIS 105, 322-328.

Gisselsson, D., Bjork, J., Hoglund, M., Mertens, F., Dal Cin, P., Akerman, M., Mandahl, N., 2001. Abnormal nuclear shape in solid tumors reflects mitotic instability. Am. J. Pathol. 158 (1), 199-206.
Gleason, D.F., 1966. Classification of prostatic carcinomas. Cancer Chemother. Rep. 50 (3), 125-128.

Hurwitz, M.D., DeWeese, T.L., Zinreich, E.S., Epstein, J.I., Partin, A.W., 1999. Nuclear morphometry predicts disease-free interval for clinically localized adenocarcinoma of the prostate treated with definitive radiation therapy. Int. J. Cancer 84 (6), 564-567.

Ikeguchi, M., Sato, N., Hirooka, Y., Kaibara, N., 1998. Computerized nuclear morphometry of hepatocellular carcinoma and its relation to proliferative activity. J. Surg. Oncol. 68, 225-230.

Ikeguchi, M., Oka, S., Saito, H., Kondo, A., Tsujitani, S., Maeta, M., Kaibara, N., 1999 Computerized nuclear morphometry: a new morphologic assessment for advanced gastric adenocarcinoma. Ann. Surg. 229, 55-61.

Kavantzas, N., Agapitos, E., Lazaris, C.H., Pavlopoulos, P.M., Sofikitis, N., Davaris, P., 2001. Nuclear/nucleolar morphometry and DNA image cytometry as a combined diagnostic tool in pathology of prostatic carcinoma. J. Exp. Clin. Cancer Res. 20 (4), 537-542.

Khan, M.A., Walsh, P.C., Miller, M.C., Bales, W.D., Epstein, J.I., Mangold, L.A., Partin, A.W., Veltri, R.W., 2003. Quantitative alterations in nuclear structure predict prostate carcinoma distant metastasis and death in men with biochemical recurrence after radical prostatectomy. Cancer 96 (12), 2583-2591.

Kontak, J.A., Campbell, S.C., 2003. Prognostic factors in renal cell carcinoma. Urol. Clin. North Am. 30 (3), 467-480.

Li, L.X., Crotty, K.A., Palmer, A.A., Kril, J.J., Scolyer, R.A., Thompson, J.F., McCarthy, S.W., 2003. Argyrophilic staining of nucleolar organizer region count and morphometry in benign and malignant melanocytic lesions. Am. J. Dermatopathol. 25 (3), 190-197.

Maiolino, P., Restucci, B., Papparella, S., De Vico, G., 2002. Nuclear morphometry in squamous cell carcinomas of canine skin. J. Comp. Pathol. 127, 114-117.

Maiolino, P., Restucci, B., Papparella, S., Paciello, O., De Vico, G., 2004. Correlation of nuclear morphometric features with animal and human world health organization international histological classifications of canine spontaneous seminomas. Vet. Pathol. 41, 608-611.

Maiolino, P., Cataldi, M., Paciello, O., Restucci, B., De Vico, G., 2005. Nucleomorphometric analysis of canine cutaneous mast cell tumours. J. Comp. Pathol. 133, 209-211.

Martano, M., Damiano, S., Restucci, B., Paciello, O., Russo, V., Maiolino, P., 2006. Nuclear morphometry in canine acanthomatous ameloblastomas and squamous cell carcinomas. Eur. J. Histochem. 50 (2), 125-130.

Martinez-Jabaloyas, J.M., Ruiz-Cerda, J.L., Hernandez, M., Jimenez, A., Jimenez-Cruz, F., 2002. Prognostic value of DNA ploidy and nuclear morphometry in prostate cancer treated with androgen deprivation. Urology 59 (2), 715-720.

Melia, J., Moseley, R., Ball, R.Y., Griffiths, D.F., Grigor, K., Harnden, P., Jarmulowicz, M., McWilliam, L.J., Montironi, R., Waller, M., Moss, S., Parkinson, M.C., 2006. A UKbased investigation of inter- and intra-observed reproducibility of Gleason grading of prostatic biopsies. Histopathology 48 (6), 644-654.

Mohler, J.L., Metts, J.C., Zhang, X.-Z., Maygarden, S.J., 1994. Nuclear morphometry in automatic biopsy and radical prostatectomy specimens of prostatic carcinoma. A comparison. Anal. Quant. Cytol. Histol. 16 (6), 415-420.

Palmieri, C., Grieco, V., 2015. Proposal of Gleason-like grading system of canine prostate carcinoma in veterinary pathology practice. Res. Vet. Sci. 103, 11-15.

Palmieri, C., Lean, F.Z., Akter, S.H., Romussi, S., Grieco, V., 2014. A retrospective analysis of 111 canine prostatic samples: histopathological findings and classification. Res. Vet. Sci. 97 (3), 568-573.

Partin, A.W., Steinberg, G.D., Pitcock, R.V., Wu, L., Piantadosi, S., Coffey, D.S., Epstein, J.I., 1992. Use of nuclear morphometry, Gleason histologic scoring, clinical stage, and age to predict disease-free survival among patients with prostate cancer. Cancer 70 (1), 161-168.

Roels, S.L.M.F., Van Daele, A.J., Van Marck, E.A., Ducatelle, R.V.A., 2000. DNA ploidy and nuclear morphometric variables for the evaluation of melanocytic tumors in dogs and cats. AJVR 61 (9), 1074-1079.

Serratosa, J., Domingo, J., Enrich, C., Bachs, O., 1988. Nuclear growth and chromatin relaxation-condensation cycle in hepatocytes during the proliferative activation of rat liver. Virchows Arch. B Cell Pathol. Incl. Mol. Pathol. 55 (1), 57-64.

Simeonov, R., Simeonova, G., 2007. Computerized cytomorphonetric analysis of nuclear area, nuclear perimeter and mean nuclear diameter in spontaneous canine mammary gland tumors. Vet. Res. Commun. 31, 553-558.

Simeonov, R., Simeonova, G., 2008. Quantitative analysis in spontaneous canine anal sac gland adenomas and carcinomas. Res. Vet. Sci. 85, 559-562.

Sorensen, F.B., 1992. Quantitative analysis of nuclear size for objective malignancy grading: a review with emphasis on new, unbiased stereologic methods. Lab. Investig. 66 (1), 4-23.

Strefezzi, R., Xavier, J.G., Catao-Dias, J.L., 2003. Morphometry of canine cutaneous mast cell tumors. Vet. Pathol. 40, 268-275.

Veltri, R.W., Marlow, C., Khan, M.A., Miller, M.C., Epstein, J.I., Partin, A.W., 2007. Significant variations in nuclear structure occur between and within Gleason grading patterns 3, 4, and 5 determined by digital image analysis. Prostate 67 (11), $1202-1210$.

Voges, G.E., McNeal, J.E., Redwine, E.A., Freiha, F.S., Stamey, T.A., 1992. The predictive significance of substaging stage A prostate cancer (A1 versus A2) for volume and grade of total cancer in the prostate. J. Urol. 147, 858-863.

Zhang, Y.H., Kanamaru, H., Oyama, N., Miwa, Y., Suzuki, Y., Akino, H., Noriki, S., Okada, K., 1999. Comparison of nuclear morphometric results between needle biopsy and surgical specimens from patients with prostate cancer. Urology 54 (4), 763-766.

Zhang, Y.H., Kanamaru, H., Oyama, N., Miwa, Y., Suzuki, Y., Akino, H., Noriki, S., Okada, K., 2000. Prognostic value of nuclear morphometry on needle-biopsy from patients with prostate cancer: is volume-weighted mean nuclear volume superior to other morphometric parameters? Urology 55, 377-381. 